\title{
ONCONET - A DIGITAL SERVICE FOR REMOTE MONITORING AND SUPPORT OF CANCER PATIENTS ON CHEMOTHERAPY
}

\author{
Irina G. Kargalskaja ${ }^{1}$, Sergei A. Shinkarev ${ }^{2}$, Boris V. Zingerman ${ }^{3}$, Inna A. Fistul ${ }^{3}$, \\ Arkadiy V. Nozik ${ }^{3}$ and Sergei B. Arseniev ${ }^{4}$ \\ ${ }^{\text {I} C o m m i t t e e ~ " P a t i e n t ~ O r i e n t e d ~ T e l e m e d i c i n e ", ~ 109390, ~ M o s c o w, ~ Y u n i h ~ L e n i n t s e v ~ 12, ~ R u s s i a n ~ F e d e r a t i o n ~}$ \\ ${ }^{2}$ Lipetsk Oncological Hospital, 398005, Lipetsk, Admiral Makarov, 1-e, Russian Federation \\ ${ }^{3}$ TelePat LLC, 115533 Moscow, Andropov Avenue 22, Nagatinsky Business Center, Russian Federation \\ ${ }^{4}$ Clinical and Research Institute for Emergency Pediatric Surgery and Trauma (CRIEPST) \\ 119180, Moscow, Bolshaya Polyanka 22, Russian Federation
}

\begin{abstract}
Background: Cancer patients suffer from many complications caused by both the disease itself and the side effects of anticancer treatment. Many symptoms go unnoticed by doctors because of the patient's remote location, significant interruptions between face-to-face visits to the doctor and differences in the individual course of the disease.In a pandemic caused by Covid 19, cancer patients are at great risk. The practical impossibility of patient's visit to a doctor, conduct clinical trials determines the highest risk for this group of patients. Thus the relevance of remote support for these patients in isolation increases many times. Objective: The goal was to create and implement in a pilot project a digital service (ONCONET system) for remote monitoring of cancer patients outside the hospital as well as adaptation to COVID 19 pandemic. Methods: The developed platform is a cloud service hosted in a data processing center which provides healthcare organizations with a ready-made service. The system is based on specialized questionnaires developed by oncologists for remote assessment of the patient's condition. The important component of the system are content libraries for patients and doctors, information and reference materials as the collection of video-schools from leading experts of nosology, rules of life, nutrition, rehabilitation and care. Results: To evaluate the effectiveness of the ONCONET system, 2 groups of patients were formed: ONCONET group - 206 patients and retrospective control group - 209 patients. Statistical analysis revealed significant differences between groups as a whole, as well as between individual factors of complications. Conclusion: The study demonstrates the usefulness and high medical efficiency of the ONCONET system for remote monitoring of cancer patients, especially against the background of a pandemic and the necessary self-isolation of cancer patients.
\end{abstract}

\section{KEYWORDS}

Remote Monitoring, Cancer Patients, Telemedicine, Tele-Rehabilitation, COVID 19

\section{INTRODUCTION}

Cancer patients suffer from many complications caused by both the disease itself and the side effects of anticancer treatment. Many symptoms go unnoticed by doctors because of the patient's remote location, significant interruptions between face-to-face visits to the doctor and differences in the individual course of the disease. Obviously it is the result of inadequate communication between a doctor and a patient. (Reilly et al., 2013). In general, worsening conditions, missed increase in symptoms indicate cancer progression or serious adverse events and cause a decrease in quality of life and survival rates (Trajkovic-Vidakovic et al., 2012).

Recently, in the United States and Europe, digital tools have been increasingly used, involving patients in collecting additional information about their condition and transmitting it electronically to the attending physician, which allows for timely and continuous collection and analysis of automatic data on the well-being of a patient outside medical facility (Jensen et al., 2014). 
If such a collection of typical symptoms is also accompanied by the possibility of automatic emergency notifications ("alarms") sent in dangerous situations to the patient and his doctor, this gives the patient a chance to respond to the problem in time, contact the doctor and reduce the possibility of developing urgent conditions or on time call an ambulance. Currently, there is convincing evidence of the effectiveness of such monitoring (Basch et al., 2017).

In a pandemic caused by Covid 19, cancer patients are at great risk. The practical impossibility of patient's visit to a doctor, conduct clinical determines the highest risk for this group of patients. Thus the relevance of remote support for these patients in isolation increases many times.

The aim of this work was to develop the ONCONET digital service for remote monitoring of cancer patients outside the hospital, as well as its pilot implementation and adaptation to the conditions of the COVID 19 pandemic.

\section{MATERIALS AND METHODS}

For remote monitoring of patients at various stages of cancer treatment, a specialized ONCONET platform developed by Telepat LLC was used (https://telepat.online/).

\subsection{ONCONET Technology Platform}

The ONCONET platform is a cloud service hosted in a data processing center and provides healthcare organizations with a ready-made service. A medical provider is registered on the platform, receives a management cabinet through which the organization registers its doctors, and each doctor then in his personal account connects his patients to monitoring process.

Technically, the platform consists of the content management module, an interactive services module, a questionnaire management and analytical modules and administrative control module.

The content management module of the platform consists of the doctor's and patient's libraries. The doctor's library includes reference, methodological and other materials useful for doctors. The patient's library contains 1,250 pages of patient-oriented content and more than 230 videos of patient schools from leading experts of nosology, rules of life, nutrition, rehabilitation and care.

The interactive service module consists of personal accounts of doctors and patients, through which they interact with the ONCONET system.

The questionnaire management module provides a centralized mechanism for electronic distribution of questionnaires as well as a special tool for designing questionnaires, with which you can quickly create new questionnaires and new monitoring schemes offered by oncologists.

One of the most important element of the system is the analytical module, which ensures the construction of so-called "heat maps" of the patient's condition and ranking patients monitored by a doctor according to the severity and urgency of the necessary attention. "Heat map" is a clear color-graphic tool for a quick assessment by the doctor of the severity and dynamics of the patient's condition "at one glance". Answering the questionnaire, the patient chooses the color of the answer (from green to red), depending on the degree of a particularsymptom. All patient's responses are displayed in a table with the observation dates vertically and the controlled symptoms horizontally. Each cell indicates the "color" of the condition selected by the patient when answering the question or selected patient's score.

\subsection{Questionnaires}

The ONCONET system is based on specialized questionnaires developed by oncologists for remote assessment of the patient's condition. One of the main problems in compiling the questionnaires was the conversion of assessments into a language that was understandable to the patient. Any criteria that the patient cannot communicate were excluded from the questionnaires. When developing questionnaires, representatives of patient communities were involved to assess their comprehensibility. Clarity of questionnaires was tested on large groups of patients. 
It is important to note that with the onset of the COVID 19 pandemic and the particular vulnerability of patients undergoing chemotherapy, a COVID 19 symptom questionnaire was added to the system. By a special mailing list, the doctors were notified of the possibility of connecting the COVID 19 questionnaire to their patients. Groups of questionnaires and number of questions are presented in Table 1.

Table 1. Groups of questionnairesand number of questions

\begin{tabular}{ll}
\hline Symptoms/Topic & $\begin{array}{l}\text { Number of } \\
\text { questions }\end{array}$ \\
\hline Covid 19 & 11 \\
Drug therapy & 109 \\
Colorectal cancer & 51 \\
Additional questionnaires & 51 \\
Mental health monitoring & 56 \\
Monitoring of surgical complication & 198 \\
Total & 476 \\
\hline
\end{tabular}

There are 2 possible variants of answers: descriptive response options, ranking from normal (marked in green) to critical (marked in red) and as a score from 0 to 10 (selected by the patient using a slider). By default, all response values are set to normal (green).However, if the patient felt problems due to any of the symptoms, then he could open this question and indicate the value that most closely matches his condition. This approach was chosen to exclude self "overdiagnosis" by patients.

\subsection{ONCONET Functional Description}

The attending physician registers the patient in the remote monitoring system, assigning him a set of specialized questionnaires and the frequency (calendar) of sending them to him. The patient receives an e-mail link to the questionnaire in accordance with the schedule set by the doctor, and answers questions in it. The questionnaires are adapted to mobile phones and are patient-oriented, accessible to the patient regardless to their level of education).

After completing the entire questionnaire, the patient receives a set of recommendations in the form of links to information materials or video schools posted on the same ONCONET portal. These materials contain tips on care and lifestyle management to reduce the impact of non-critical symptoms reported by the patient. If the patient indicated a dangerous or critical symptom, he will immediately receive a notification "Call an ambulance immediately!" or "Contact your doctor Immediately!".

The system also allows the patient to attach additional documents for example, analyses performed in another laboratory, and write a message to the doctor describing a symptom or problem not included in the questionnaire. In addition to the questionnaires, the doctor can also assign the patient one or more times a day to monitor physiological parameters (pressure, temperature, glucose, weight), as well as reminders about taking medications, with confirmation of reception.

The results of the comprehensive monitoring are sent to the remote cloud server for automatic processing and then sent to the doctor in the form of a so-called "heatmap" showing the dynamics of the patient's symptoms. Periodically (according to the established schedule), the doctor also receives a summary of all his patients who are on remote monitoring. The summary is ranked by the overall complex severity of the patient's symptoms, so that the doctor can more quickly assess the condition of the most severe patients and take appropriate measures.

If necessary, the doctor can write an additional recommendation to the patient directly in the ONCONET system, invite them to a face-to-face appointment, or send a link to additional material with recommendations.

\subsection{Clinical Study}

A pilot project for the implementation of the ONCONET system was conducted in 2018-2019 in the Lipetsk region, in the Regional Oncology Hospital. The project involved 61 doctors and 206 patients on remote monitoring. Patients with breast cancer, lung cancer, cervical and ovarian cancer, prostate, thyroid, kidney, stomach and colorectal cancer and Hodgkin's lymphoma were included in the study. 
To evaluate the effectiveness of the ONCONET system, 2 groups of patients were formed: the ONCONET group - 206 patients and theretrospective control group - 209 patients. The groups did not differ statistically in terms of diagnoses and stages of diseases, treatment protocols used, gender, and age.

The groups were compared by the frequency of complications and adverse reactions during treatment, as well as the most important indicator - the transfer or postponement of the next course of chemotherapy.

Chi-square values, two-sided, statistical difference between frequencies were calculated. A statistically significant difference was considered at $\mathrm{p}<0.05$. Statistical analysis was performed using the STATISTICA 8 package.

\section{RESULTS AND DISCUSSION}

The results of comparing the groups are presented in Table 2.

Table 2

\begin{tabular}{llll}
\hline $\begin{array}{l}\text { Complications/ } \\
\text { Chemotherapy date } \\
\text { change }\end{array}$ & ONCONET group \% & Reference group \% & Significance \\
\hline Hematological & 32.5 & 41.6 & \\
Hepatotoxic & 29.1 & 23.0 & $\mathrm{p}=0.0227(<0.05)$ YES \\
$\begin{array}{l}\text { Nephrotoxic } \\
\text { Total }\end{array}$ & 3.4 & 13.4 & $\mathrm{p}=0.1022(>0.05)$ NO \\
$\begin{array}{l}\text { Chemotherapy date } \\
\text { change }\end{array}$ & 7.8 & 78 & $\mathrm{p}=0.001(<0.05) \quad$ YES \\
& & 12.9 & $\mathrm{p}=0.0177(, 0.05) \quad$ YES
\end{tabular}

The total number of the three most significant complications decreased by $13 \%$ (65 vs 78 ), hematological complications significantly decreased by $9 \%$ (32.5 vs 41.6), and the number of nephrotoxic complications decreased by $11 \%$ (3.4 vs 13.4 ).

There were no significant differences between the groups in hepatotoxic complications. Moreover, the percentage of these complications was higher in the ONCONET group (29\% vs 23\%). This phenomenon is probably associated with a more sensitive diagnosis of hepatotoxic abnormalities in the ONCONET system, due to adequate questionnaires and the methodology of their application for monitoring.

Among other less quantitative complications, it should be noted a 3-times reduction in gastrointestinal disorders ( $0.7 \%$ vs. $2.6 \%)$, neurotoxic and skin manifestations are practically minimized.

One of the most important results is a statistically significant decrease, almost one and a half times, of the postponement of the date of the next course of chemotherapy (7.9\% vs $11.9 \%)$ in the ONCONET group. As a result, this made it possible to maintain the necessary intervals between courses of chemotherapy, and as a result, improve survival rates.

In total, the ONCONET system was tested during 2018-2020 in 22 medical organizations in 10 regions of Russia. The system was used by 174 doctors and 382 patients. Initially, 460 patients were connected to the system but only $382(92 \%)$ out of them actually participated in the monitoring. This indicates a high level of demand for the system by patients. A total of 21936 questionnaires were completed by patients at the time of publication (an average of 57 per patient) and 193248 symptoms were reported (more than 500 on average per patient).

The use of heatmaps and algorithms for ranking patients according to the severity of the condition enable the doctor to quickly respond to negative dynamics and thereby increase patient safety. In the current version of the ONCONET system, estimation algorithms based on weight coefficients proposed by oncologists are used, however, further development of these algorithms based on machine learning is also planned in the future.

Doctor's and a patient's libraries are of independent value. These informational and reference materials and video schools are constantly being developed and can be used not only within the ONCONET system, but also in any other means of content delivery. 
Patient questionnaires are also an essential part of the system. Not only oncologists, but also psychologists and linguists participated in the development of the questionnaires, whose task was to make the questionnaires simple and understandable for a wide range of patients, while preserving their diagnostic value.

The addition of the COVID 19 block was due to the corona virus pandemic. Undoubtedly, this block remains relevant and in demand even after the end of the pandemic. The COVID 19 questionnaire, when further developed, can serve as an independent tool for remote rehabilitation of patients who have patients who have undergone the infection.

\section{CONCLUSION}

This study demonstrates the unconditional usefulness and high medical efficiency of remote monitoring of cancer patients, especially against the background of a pandemic and the necessary self-isolation of cancer patients. Remote monitoring and doctor support is in demand by cancer patients. This significantly improves the quality of life, satisfaction with the treatment, loyalty and adherence to the treatment, maintaining the right lifestyle recommended by the doctor, which also contributes to the increase in the effectiveness of rehabilitation.

It is extremely important to make the provision of remote monitoring services to cancer patients free of charge. This requires the full support of the state and local authorities, as well as public and non-profit organizations. It is also necessary to encourage oncologists and medical organizations to widely implement this service.

The developed ONCONET system is a universal platform, which, through appropriate content filling, can be adapted for other areas of distance rehabilitation. Currently, work is underway to create a system for remote monitoring of children with the consequences of a craniocerebral and spinal injury "Kids-rehab".

\section{ACKNOWLEDGEMENT}

The authors are deeply grateful to the oncologists of the Lipetsk region, as well as to all cancer patients who participated in the ONCONET pilot project.

\section{REFERENCES}

Basch E. et al, 2017, Overall survival results of a trial assessing patient-reported outcomes for symptom monitoring during routine cancer treatment, JAMA, Vol. 318, No. 2, pp 197-198.

Jensen R.E. et al, 2014, Review of electronic patient-reported outcomes systems used in cancer clinical care, J Oncol Pract Vol. 10, No 4, pp e215-e222.

Reilly C. M. et al, 2013 A literature synthesis of symptom prevalence and severity in persons receiving active cancer treatment, Support Care Cancer, Vol. 21, No. 6, pp1525-1550.

Trajkovic-Vidakovic M. et al, 2012, Symptoms tell it all: a systematic review of the value of symptom assessment to predict survival in advanced cancer patients, Crit Rev Oncol Hematol, Vol. 84, No 1, pp130-148.

https://telepat.online/ 